\title{
Arylation of Diethyl AlkyImalonates: Synthetic Route to Diethyl Alkyl(substituted Aryl)malonates with the Aid of Temporary Arene Complexation by the Cyclopentadienyliron Moiety
}

\author{
Adam Piórko, Alaa S. Abd-El-Aziz, Choi Chuck Lee, ${ }^{*}$ and Ronald G. Sutherland * \\ Department of Chemistry, University of Saskatchewan, Saskatoon, Saskatchewan, S7N OWO Canada
}

\begin{abstract}
Nucleophilic substitution of chlorine in the ironcyclopentadienyl hexafluorophosphates of chlorobenzene, isomeric chlorotoluenes and dichlorobenzenes (1) with the anions generated from diethyl alkylmalonates (2) and (3) leads to the formation of ironcyclopentadienyl complexes of diethyl alkyl(substituted phenyl)malonates (4) and (5). An excess of the anion employed in reactions with the $m$ - and $p$-dichlorobenzene complexes leads to substitution of both chlorine atoms and formation of isomeric phenylenedimalonate complexes (8). Malonyl cations (4) and (5) possessing a chloro substituent on the complexed phenyl ring provide the possibility of further modifications of phenyl ring substituents via substitution [complex (10)] or addition reactions [complexes (12)]. The described complexes have been demetallated giving diethyl alkyl(substituted phenyl)malonates in $>50 \%$ overall yield from the cations (1). All the complexes and compounds have been fully characterized. This approach to the synthesis of the diethyl alkyl(substituted phenyl)malonates which are intermediates in the synthesis of important biologically active barbiturates is easy, efficient, and currently the most general in the area.
\end{abstract}

5-Ethyl-5-phenylbarbituric acid (phenobarbital) is an important drug used widely as a central nervous system (CNS) agent, as an enzyme inducer, and as one of the most efficient drugs in the treatment of epilepsy. ${ }^{1-4}$ The usual method of synthesis involves condensation of phenylmalonyl esters with urea. An alkyl substituent which will occupy position 5 of the target acid may be introduced prior to or after the condensation with urea and the first possibility is usually employed. It has been established that the phenyl substituent must be introduced into the malonyl ester prior to an alkyl one. ${ }^{1}$ This requirement is satisfied in all previously described syntheses of alkyl(phenyl)malonyl esters. The usual preparation of phenylmalonyl esters utilizes a Claisen condensation of phenylacetic acid esters with a dialkyl oxalate followed by decarbonylation of the resulting keto diester to phenylmalonate esters. ${ }^{1,2}$ It was first noted by Nelson and Cretcher ${ }^{5}$ that, due to problems with the synthesis of (substituted phenyl)acetic acids, only a few examples of 5ethyl-5-(substituted phenyl)barbiturates have been described and their list, as presented by Doran, ${ }^{6}$ is rather short. A recent report by Carrisimi et al. ${ }^{7}$ on the synthesis of some 5-ethyl5-(halogenophenyl)barbiturates also shows that the overall efficiency of such synthesis is low. In recent years $\pi$-complexes of arenes with various metals have been studied with the aim of applying them in preparative organic chemistry. Most of the earlier studies involved (arene)chromium(tricarbonyl) complexes $^{8,9}$ with both nucleophilic addition and substitution reactions being thoroughly investigated. The synthesis of phenylmalonate with the use of chromium tricarbonyl complexes of chloro- or fluoro-benzene has been reported ${ }^{10}$ but no practical applications have followed to date. Recently a number of reports describing the use of (halogenoarene)iron(cyclopentadienyl) complexes in nucleophilic substitution reactions with carbon nucleophiles leading to (substituted phenyl)malonates appeared ${ }^{1-14}$ and this has now been followed by the description of such complete syntheses under mild conditions. ${ }^{15}$ The route involves arylation of diethyl malonate with various disubstituted arenes with one of the substituents being a chloro group, followed by the thermal demetallation of the (substituted phenyl)malonate complexes. Since this method requires that all the phenyl substituents' modifications be completed before the arylation step ${ }^{12,15}$ we have extended its range by the use of diethyl alkylmalonates instead of malonates in nucleophilic substitution reaction.

\section{Results and Discussion}

Reactions of cations (1a-f) with diethyl alkylmalonates (2) and (3) have been carried out under very mild conditions upon the addition of a mixture of malonate and potassium t-butoxide in tetrahydrofuran (THF) to an equimolar (with malonate) solution of cation (1) in dimethylformamide (DMF). Gentle reflux of that mixture led to the conversion of cation (1) into the product (4) or (5) in a nucleophilic substitution reaction of the complexed chloroarene with the carbanion generated from alkylmalonate (Scheme 1).

Cyclopentadienyliron complexes of diethyl alkyl(substituted phenyl)malonates $(\mathbf{4 c}-\mathbf{f})$ and $(\mathbf{5 a}, \mathbf{b}$, and $\mathbf{f})$ which are the products of these reactions were easily isolated and purified by column chromatography. Use of excess of diethyl alkylmalonate in reactions with dichlorobenzene complexes (1e) and (1f) leads to contamination of the product $(\mathbf{4 e}),(\mathbf{4 f}),(\mathbf{5 f})$ due to disubstitution. Cationic product complexes are solidifying oils at room temperature and we were unable to crystallize them despite many attempts. Crystals were obtained in the case of cations (4c) and (4d) from ethanol at $-18{ }^{\circ} \mathrm{C}$; however, they melted at room temperature. Identity of the products was confirmed by analytical and i.r. ${ }^{16}$ data which are presented in the Experimental section. Chemical shifts and splitting patterns of both proton and carbon signals observed in high-resolution ${ }^{1} \mathrm{H}$ and ${ }^{13} \mathrm{C}$ spectra are in full agreement with expectations. Also, integration of various signals confirmed the presence of the expected number of protons or carbons. It may be noted that for the cationic products with substituents located in both ortho and meta positions [(4d), (4e), and (5b)], steric hindrance effects were observed. Thus in the proton spectra of these complexes, the usual triplets and quartets for the methyl and methylene protons of the ethyl ester groups became multiplets, and in the ${ }^{13} \mathrm{C}$ spectra two signals were observed for the carbonyl, methyl and methylene carbon atoms.

Demetallation of cationic complexes in pyrolytic sublimation $\left(200-230^{\circ} \mathrm{C} ; 13 \mathrm{~N} \mathrm{~m}^{-2}\right)$ has been used effectively for liberation of arenes and has recently been described in the synthesis of 


$$
\begin{aligned}
& R=F e^{I}(c p) P F_{6} \\
& R^{2}
\end{aligned}
$$

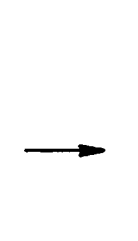

(2) $\mathrm{R}^{1}=\mathrm{Me}$

(3) $\mathrm{R}^{1}=\mathrm{Et}$<smiles>[R][R]c1ccc(C([R])(C(=O)OCC)C(=O)OCC)cc1</smiles>

$(4 c-f) R^{\prime}=M e$

$(5 a, b, f) \quad R^{\prime}=E t$<smiles>[R][R]c1ccc(C([R])(C(=O)OCC)C(=O)OCC)cc1</smiles>

$(6 c-f) R^{\prime}=M e$

$(7 a, b, f) R^{1}=E t$

Scheme 1.<smiles>[R]CC1CCCCC1[R]</smiles>

(1e) $\mathrm{R}^{2}=m-\mathrm{Cl}$

(1f) $\mathrm{R}^{2}=p-\mathrm{Cl}$<smiles>CCOC(=O)C(CC)C(=O)OCC</smiles>

(3)<smiles>[R]COC(=O)C(CC)(CC)C(CC)(CC)c1cccc(C(CC)(C(=O)OCC)C(=O)OCC)c1</smiles>

(8e) meta

(8f) para<smiles>CCOC(=O)C(CC)(C(=O)OCC)C(CC)(C(=O)OCC)c1ccccc1</smiles>

(9e) meta

(9f) para

Scheme 2.

arylmalonates. ${ }^{15}$ That method has been successfully applied in the present work, giving diethyl alkyl(substituted phenyl)malonates $(\mathbf{6 c}-\mathbf{f})$ and $(\mathbf{7 a}, \mathbf{b}$, and $\mathbf{f})$ from the respective cations in $72-80 \%$ yield. While some of them have been characterized previously, the analytical results for the new compounds as well as the mass spectra and high-resolution n.m.r. spectra for all the compounds obtained are presented in the Experimental section. The only significant difference in the n.m.r. spectra of cations and metal-free compounds comes from the disappearance of cyclopentadienyl signals and shifts of aromatic signals toward lower fields.

When the amounts of malonate (3) and potassium tbutoxide used in the reaction were increased, substitution of both chlorine atoms in cations (1e) and (1f) was observed, leading to the formation of isomeric $m$ - and $p$-phenylenedimalonate complexes (8e) and (8f), respectively (Scheme 2). The structures of these complexes have been unambiguously established on the basis of analytical data, presented in the Experimental section. Owing to the symmetry of the molecules, the alkyl protons and the carbon signals of the side chains of (8e) and (8f) show, in the integration, double the number of protons and carbons as compared with cations (4) and (5). Also in the ${ }^{13} \mathrm{C}$ spectra, the lower-field signal of the quaternary carbon of an aromatic ring bearing the chloro substituent disappeared and the high-field quaternary aromatic carbon signal shows the presence of two identical carbon atoms in the molecule. These complexes have been demetallated as described above yielding the previously unknown isomeric $m$ and $p$-phenylenedimalonate (9e) and (9f), respectively. Their structures have also been confirmed by analytical data. Attempts to prepare the analogous ortho isomer using both malonates (2) and (3) were unsuccessful. Among the products of such reactions carried out even for extended reaction times were the monosubstitution product and the complex of ethyl (o-chlorophenyl)acetate as established by their n.m.r. spectra. The second product results from the cleavage of the monosubstitution product in the presence of base as indicated in the literature. ${ }^{17}$ It is likely that in the case of the ortho-disubstituted substrate, steric hindrance of the first introduced malonyl substituent prevents the introduction of the second group.

It is interesting to note that our synthesis of complexes (8e) and (8f) involves the first reported double nucleophilic substitution reaction on metal-arene complexes employing carbon nucleophiles. In the previous studies only monosubstitution products have been reported when nitrogen or carbon nucleophiles were used. ${ }^{12,15}$ For the first time, however, a tertiary carbanion has been employed in such reactions and, as discussed below, the mechanism of the reaction allows the substitution of the second chlorine on the phenyl ring in such a situation.

In the case of the previously reported complexes of (substituted phenyl)malonates, ${ }^{15}$ further modifications of the phenyl substituents or the subsequent substitution of the second chlorine could not be achieved. Under basic conditions, deprotonation of the methine proton of the malonyl group would lead to the formation of a cyclohexadienyl complex with an exocyclic double bond ${ }^{12,15}$ and this complex would be unreactive in both nucleophilic substitution and addition reactions. Thus we were unable to methylate these complexes using the procedures widely applied for that purpose ${ }^{18}$ when either a malonyl or a methyl substituent of the complexed arene ring was involved. This is not the case with the alkyl(phenyl)malonates studied in the present work where a quaternary carbon atom is bonded to the complexed aromatic ring and we were able to modify the substituents on the phenyl ring further using nucleophilic substitution or addition reaction (Scheme 3). 
<smiles>CCOCC(C)(C(=O)OCC)c1ccccc1</smiles>

(4e)<smiles>CCOC(=O)C(C)(C(=O)OCC)c1cccc(C(C)C)c1</smiles><smiles>CCOC(=O)C(C)(C(=O)OCC)c1ccccc1Cl</smiles>

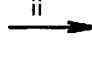<smiles>CCOC(=O)C(C)(C(=O)OCC)c1cccc(N2CCOCC2)c1</smiles>

(10)

(12a)<smiles>CCOC(=O)C(C)(C(=O)OCC)c1ccc(C(C)C)cc1</smiles>

(12b)<smiles>CCOC(=O)C(C)(C(=O)OCC)c1cccc(N2CCOCC2)c1</smiles>

(11)<smiles>CCOC(=O)C(C)(C(=O)OCC)c1ccccc1Cl</smiles>

(13a)<smiles>CCOC(=O)C(C)(C(=O)OCC)c1ccccc1</smiles>

(13b)

$P=F e^{I I} c p$

Scheme 3. Reagents: i, morpholine; ii, $\mathrm{NaCN}$

Thus reaction of the complex (4e) with morpholine under conditions used previously ${ }^{12}$ gave the diethyl methyl $(m$ morpholinophenyl)malonate complex (10) which upon demetallation yielded free ligand (11). The analytical data for both the complex and free ligand confirm their structures. In the proton spectra of both compounds two four-proton multiplets of the morpholine part appear, while in their ${ }^{13} \mathrm{C}$ spectra two two-carbon signals of the morpholine skeleton are observed. In addition, the signals of the aromatic quaternary carbons bearing a chloro substituent present in both complex (4e) and the liberated arene (6e) disappeared and the new signals of the quaternary aromatic carbons bonded to nitrogen are observed.

A second method of modification of the complexed phenyl ring employing a nucleophilic addition reaction was also studied. Using conditions described previously for addition of the cyano anion to activated complexes ${ }^{19,20}$ we have examined this reaction with cation (4d) as a substrate. A mixture of two isomers resulting from cyano addition ortho and meta to the chloro substituent in the complexed ring, (12a) and (12b), respectively, in the ratio $6: 1$, was obtained. The structures of these products were established on the basis of analytical results and their n.m.r. spectra ${ }^{19-21}$ (see Experimental section) even if the isomers were not separated. Unlike prior studies with the $o$ chlorotoluene complex, a meta adduct was formed as well as the ortho one, and this may be due to the electronic effect (different from that of the methyl group) and steric effect of the bulky malonate substituent. It is also possible that the stability of the meta adduct favours its formation.

The mixture of isomeric adducts (12a) and (12b) was efficiently demetallated employing the 2,3-dichloro-5,6-dicyano- p-benzoquinone (DDQ) procedure, ${ }^{22}$ yielding a mixture of compounds (13a) and (13b) in the ratio 4:1. Analytical data confirm the structures of the demetallated products, and their ratio was estimated from the integration of signals in a ${ }^{13} \mathrm{C}$ spectrum registered in an inverse gated decoupling experiment.

The results obtained in the course of this study seem to remove one of the current barriers in the synthetic chemistry of phenylbarbiturates. Usually, introduction of a substituent into the phenyl ring of a phenylbarbiturate was completed in one of two ways: (i) at any stage preceding the condensation of the dialkyl alkyl(phenyl)malonate with urea, or (ii) through reactions of the phenyl ring of the alkyl(phenyl)barbituric acid. The first method involves the preparation of (substituted phenyl)acetic acid esters which are next condensed with a dialkyl oxalate or a dialkyl carbonate to yield the dialkyl alkyl(substituted phenyl)malonate ${ }^{7,17,23-25}$ or the introduction of the substituent into the phenyl ring of the dialkyl alkyl(phenyl)malonate. ${ }^{5}$ In both cases the overall yields of dialkyl alkyl(substituted phenyl)malonates are relatively low because of either decreased reactivity of the (substituted phenyl)acetic ester in condensation leading to malonates as compared with the parent compound itself ${ }^{1}$ or by the low yield of functionalization of the phenyl ring in the dialkyl alkyl(phenyl)malonate. ${ }^{5} \mathrm{~A}$ similar situation has been observed in the case of direct functionalization of the phenyl ring of alkyl(phenyl)barbituric acid $^{26,27}$ investigated as an alternative route. In addition, restrictions have also been observed regarding the kind of substituent introduced to the phenyl ring.

Another approach to the synthesis of the alkyl(substituted phenyl)barbiturates has been described by Pinhey and co- 
workers. ${ }^{28,29}$ They used the reaction of barbituric or 5-ethylbarbituric acid with aryl-lead triacetates to synthesize barbiturates with a substituted phenyl ring in yields $55-91 \%$. Their study, however, involved only electron-donating substitutents (methyl or methoxy in the para position) since direct plumbylation $^{30}$ of aromatic compounds bearing electronwithdrawing substituents must be replaced with a longer route involving either arylmercury or trimethylsilyl intermediate compounds.

Recently Salomon and co-workers ${ }^{31}$ described a method involving electrophilic substitution of hydrogen in arenes bearing electron-donating substituents with diethyl oxomalonate. Diethyl aryltartronates obtained from such reactions were converted into (substituted phenyl)malonates through reductive deoxygenation but in some cases they had to be transformed into acetates prior to the deoxygenation step. Since this approach shows selectivity toward formation of para products (ortho if position para is blocked) the authors widened the scope of the reaction by using lithiation or formation of aryl Grignard reagents prior to the reaction with oxomalonate. Thus their approach, while advantageous over those previously reported, leads to ortho or para substitution, and requires that relatively demanding reaction conditions be used and that the alkylation step be completed subsequently.

After the initial reports concerning the use of arene-metal $\pi$-complexes as electrophiles in reactions with various nucleophiles, reactions of (halogenobenzene)chromium tricarbonyls with the diethyl malonate anion have been studied by Semmelhack and Hall. ${ }^{10}$ They found that the reaction of the chlorobenzene complex in hexamethylphosphoramide followed by demetallation yielded, at best, $51 \%$ of diethyl phenylmalonate with complications arising from side reactions. Higher yields were obtained from the fluorobenzene complex $(>95 \%)$. To our best knowledge this initial communication has not been followed by any other study involving chromium complexes with more complicated substitution pattern in the phenyl ring.

It is known that the ironcyclopentadienyl system activates the chlorine atom of chlorobenzene complexes in substitution reactions to a significantly higher degree than does the chromium tricarbonyl moiety, ${ }^{32,33}$ and such chloroarene complexes have been found to undergo an easy nucleophilic substitution reaction with carbanions ${ }^{11-15}$ including that derived from diethyl malonate. These reactions, followed by demetallation, yielded a number of (substituted phenyl)malonates. ${ }^{15}$ However, further modifications of the phenyl substituents under basic conditions (substitution or addition reactions) would not be possible since, in the presence of a base, immediate deprotonation of the methine carbon adjacent to the complexed ring would give rise to cyclohexadienyl complexes in which both the ring and its substituent are unreactive. ${ }^{12}$ Thus the only way a phenyl substituent could be modified is the substitution of one of the two chlorines in a dichlorobenzene complex with oxygen, sulphur, or secondary amino nucleophiles followed by a substitution of the second chlorine of the ring with the malonyl anion. Reactions reported in the present work show that replacement of diethyl malonate with diethyl alkylmalonate makes these restrictions invalid and synthesis of the compounds (9), (11), and (13) proves that point beyond any doubt.

In summary we would point out that the route we propose is preparatively easy and flexible, allowing the ready synthesis of selected products and giving the expected diethyl alkyl(substituted phenyl)malonates in good overall yields $[\sim 50 \%$ calculated on starting cations (1)]. This route is advantageous over all those described earlier and should be of practical value in the search for compounds related to 5-ethyl-5-phenylbarbiturate.

\section{Experimental}

M.p.s were measured in a capillary and are uncorrected. I.r. spectra were recorded on the neat samples (oils) or in potassium bromide discs. M.s. spectra (electron impact ionization) are reported in $m / z$ units. ${ }^{1} \mathrm{H}$ and ${ }^{13} \mathrm{C}$ N.m.r. spectra were recorded on a Bruker AM300 instrument with $\mathrm{SiMe}_{4}$ as internal standard for ${ }^{1} \mathrm{H}$ spectra; chemical shifts were calculated from the solvent signals for the ${ }^{13} \mathrm{C}$ spectra. Alumina F-20 (Alcoa Chimica) deactivated by exposure to air for $48 \mathrm{~h}$, and silica gel (Merck Co.) $70-230 \mathrm{mesh}$, were used in the column chromatographic purification of the various complexes and the liberated arenes, respectively.

Starting Materials.-Starting complexes (1a-f) were prepared as their hexafluorophosphates according to the procedures published previously. ${ }^{34}$ Diethyl methyl- and ethylmalonate as well as all the solvents were commercially available (reagent grade) and were used without further purification.

General Procedure for the Nucleophilic Substitution Reactions.-All the nucleophilic substitution reactions were completed using $3 \mathrm{mmol}$ of the starting cations (1).

A mixture obtained from a mixture of potassium t-butoxide $(0.56 \mathrm{~g}, 5 \mathrm{mmol})$ with diethyl methyl- or ethyl-malonate $(3 \mathrm{mmol})$ in THF $(50 \mathrm{ml})$ vigorously stirred for $5 \mathrm{~min}$ was added to a stirred solution of a cation (1) $(3 \mathrm{mmol})$ in DMF $(15 \mathrm{ml})$. The reaction mixture was stirred under gentle reflux for $c a .10 \mathrm{~h}$; the resulting dark brown solution with some white precipitate was cooled to room temperature and $10 \%$ hydrochloric acid $(10 \mathrm{ml})$ and solid ammonium hexafluorophosphate $(0.5 \mathrm{~g}, c a$. $3 \mathrm{mmol}$ ) were added. After being stirred for $15 \mathrm{~min}$ the yellowbrown solution was concentrated to $c a .30 \mathrm{ml}$ on a rotary evaporator and then extracted with methylene dichloride until the water layer became colourless. The combined extract was washed with water $(5 \times 20 \mathrm{ml})$, dried over magnesium sulphate, and the solvent was evaporated off under reduced pressure at $30^{\circ} \mathrm{C}$. The residual brown oil was washed with diethyl ether $(3 \times 20 \mathrm{ml})$, and then purified using column chromatography on deactivated Alumina F20 ( $5 \mathrm{~cm}$ column). The crude product was washed first on the column with carbon tetrachloride and then the product, with a small amount of the starting cation, was eluted using chloroform. The purification was repeated and the product was eluted from the column using a mixture of chloroform-carbon tetrachloride $(4: 1)$. Evaporation of the solvents from the eluate followed by drying in vacuo gave the pure product (4) or (5).

The double nucleophilic substitution products were obtained in a similar way; however, THF $(50-100 \mathrm{ml})$ was used instead of DMF and potassium t-butoxide $(10 \mathrm{mmol})$ with diethyl alkylmalonate $(7 \mathrm{mmol})$ in THF $(100 \mathrm{ml})$ were used to prepare malonyl anions. Thus were prepared:

( $\eta$-Cyclopentadienyl $)[\eta$-diethyl ethyl(phenyl)malonate $]$ iron(II) hexafluorophosphate (5a), solidifying orange-brown oil $\left(1.12 \mathrm{~g}, 70 \%\right.$ ) (Found: $\mathrm{C}, 45.1 ; \mathrm{H}, 4.6 . \mathrm{C}_{20} \mathrm{H}_{25} \mathrm{~F}_{6} \mathrm{FeO}_{4} \mathrm{P}$ requires $\mathrm{C}, 45.2 ; \mathrm{H}, 4.7 \%) ; v_{\max }$.(neat) $1730 \mathrm{~cm}^{-1}(\mathrm{CO}) ; \delta_{\mathrm{H}}\left(\mathrm{CDCl}_{3}\right) 6.6-$ $6.4(3 \mathrm{H}$, br s, ArH $), 6.35-6.20(2 \mathrm{H}, \mathrm{br} \mathrm{s}, \mathrm{ArH}), 5.10(5 \mathrm{H}, \mathrm{br} \mathrm{s}$, $\left.\mathrm{C}_{5} \mathrm{H}_{5}\right), 4.43-4.33\left(4 \mathrm{H}, \mathrm{m}, \mathrm{CO}_{2} \mathrm{CH}_{2}\right), 2.28(2 \mathrm{H}, \mathrm{q}, J 7.2 \mathrm{~Hz}$, $\mathrm{MeCH} \mathrm{C}_{2} \mathrm{C}, 1.43-1.36\left(6 \mathrm{H}, \mathrm{m}, \mathrm{CO}_{2} \mathrm{CH}_{2} \mathrm{Me}\right)$, and $0.83(3 \mathrm{H}, \mathrm{t}, \mathrm{J}$ $\left.7.2 \mathrm{~Hz}, \mathrm{MeCH}_{2}\right) ; \delta_{\mathrm{C}}\left(\left[{ }^{2} \mathrm{H}_{6}\right]\right.$ acetone $) 168.34\left(2 \mathrm{C}, \mathrm{CO}_{2}\right), 103.58$ (quaternary ArC), 88.04 (ArC), 87.60 (2 C, ArC), 86.96 (2 C, $\mathrm{ArC}), 77.82\left(5 \mathrm{C}, \mathrm{C}_{5} \mathrm{H}_{5}\right), 62.64\left(2 \mathrm{C}, \mathrm{CO}_{2} \mathrm{CH}_{2}\right), 61.18$ (quaternary aliph.), $29.95\left(\mathrm{CCH}_{2} \mathrm{Me}\right), 13.44\left(2 \mathrm{C}, \mathrm{CO}_{2} \mathrm{CH}_{2} \mathrm{Me}\right)$, and $8.85\left(\mathrm{CCH}_{2} \mathrm{Me}\right)$.

( $\eta$-Cyclopentadienyl)[ $\eta$-diethyl ethyl(o-tolyl)malonate]iron(II) hexafluorophosphate $(\mathbf{5 b})$, solidifying orange oil $(1.10 \mathrm{~g}$, 67\%) (Found: $\mathrm{C}, 46.5 ; \mathrm{H}, 5.0 . \mathrm{C}_{21} \mathrm{H}_{27} \mathrm{~F}_{6} \mathrm{FeO}_{4} \mathrm{P}$ requires $\mathrm{C}$, $46.25 ; \mathrm{H}, 5.0 \%$ ); $v_{\text {max. }}$ (neat) $1735 \mathrm{~cm}^{-1}(\mathrm{CO}) ; \delta_{\mathrm{H}}\left(\mathrm{CDCl}_{3}\right) 6.46$ $6.35(2 \mathrm{H}, \mathrm{m}, \mathrm{ArH}), 6.30(1 \mathrm{H}, \mathrm{br} \mathrm{s}, \mathrm{ArH}), 6.19(1 \mathrm{H}, \mathrm{d}, J 5.4 \mathrm{~Hz}$, 
$\mathrm{ArH}), 5.10\left(5 \mathrm{H}, \mathrm{s}, \mathrm{C}_{5} \mathrm{H}_{5}\right), 4.41\left(2 \mathrm{H}, \mathrm{q}, J 7.1 \mathrm{~Hz}, \mathrm{CO}_{2} \mathrm{CH}_{2}\right)$, $4.27-4.19\left(2 \mathrm{H}, \mathrm{m}, \mathrm{CO}_{2} \mathrm{CH}_{2}\right), 2.66-2.53(1 \mathrm{H}, \mathrm{m}, \mathrm{CCH} \mathrm{HMe})$, $2.42(3 \mathrm{H}, \mathrm{s}, \mathrm{ArMe}), 2.28-2.16(1 \mathrm{H}, \mathrm{m}, \mathrm{CCH} H \mathrm{Me}), 1.41(3 \mathrm{H}, \mathrm{t}$, $\left.J 7.1 \mathrm{~Hz}, \mathrm{CO}_{2} \mathrm{CH}_{2} \mathrm{Me}\right), 1.30-1.22\left(3 \mathrm{H}, \mathrm{m}, \mathrm{CO}_{2} \mathrm{CH}_{2} \mathrm{Me}\right)$, and $1.0-0.91\left(3 \mathrm{H}, \mathrm{m}, \mathrm{CCH}_{2} \mathrm{Me}\right) ; \delta_{\mathrm{C}}\left(\left[{ }^{2} \mathrm{H}_{6}\right]\right.$ acetone $) 169.30\left(\mathrm{CO}_{2}\right)$, $168.65\left(\mathrm{CO}_{2}\right), 105.36$ (quaternary $\mathrm{ArC}$ ), 102.59 (quaternary $\mathrm{ArC}$ ), 90.34, 88.21, 86.93, and 86.78 (ArC), $78.57\left(5 \mathrm{C}, \mathrm{C}_{5} \mathrm{H}_{5}\right.$ ), $63.18\left(\mathrm{CO}_{2} \mathrm{CH}_{2}\right), 62.91\left(\mathrm{CO}_{2} \mathrm{CH}_{2}\right), 61.47$ (quaternary aliph.), $29.61\left(\mathrm{CCH}_{2} \mathrm{Me}\right), 20.20(\mathrm{ArMe}), 13.89\left(\mathrm{CO}_{2} \mathrm{CH}_{2} \mathrm{Me}\right), 13.74$ $\left(\mathrm{CO}_{2} \mathrm{CH}_{2} \mathrm{Me}\right), 10.03\left(\mathrm{CCH}_{2} \mathrm{Me}\right)$.

( $\eta$-Cyclopentadienyl $)[\eta$-diethyl methyl(p-tolyl)malonate]iron(II) hexafluorophosphate $(\mathbf{4 c})$, orange-brown oil $(1.42 \mathrm{~g}$, $89 \%$ ) (Found: $\mathrm{C}, 45.1 ; \mathrm{H}, 4.5 . \mathrm{C}_{20} \mathrm{H}_{25} \mathrm{~F}_{6} \mathrm{FeO}_{4} \mathrm{P}$ requires $\mathrm{C}, 45.2$; $\mathrm{H}, 4.7 \%) ; v_{\max }$ (neat) $1732 \mathrm{~cm}^{-1}(\mathrm{CO}) ; \delta_{\mathrm{H}}\left(\mathrm{CDCl}_{3}\right) 6.32(2 \mathrm{H}, \mathrm{d}, J$ $5.5 \mathrm{~Hz}, \mathrm{ArH}), 6.25(2 \mathrm{H}, \mathrm{d}, J 5.5 \mathrm{~Hz}, \mathrm{ArH}), 5.01\left(5 \mathrm{H}, \mathrm{s}, \mathrm{C}_{5} \mathrm{H}_{5}\right)$, $4.34\left(4 \mathrm{H}, \mathrm{q}, J 6.8 \mathrm{~Hz}, \mathrm{CO}_{2} \mathrm{CH}_{2}\right), 2.54(3 \mathrm{H}, \mathrm{s}, \mathrm{ArMe}), 1.94(3 \mathrm{H}, \mathrm{s}$, $\mathrm{CMe})$, and $1.33\left(6 \mathrm{H}, \mathrm{t}, J 6.8 \mathrm{~Hz}, \mathrm{CH}_{2} \mathrm{Me}\right) ; \delta_{\mathrm{C}}\left(\left[{ }^{2} \mathrm{H}_{6}\right]\right.$ acetone $)$ $169.53\left(2 \mathrm{C}, \mathrm{CO}_{2}\right), 104.28$ (quaternary $\mathrm{ArC}$ ), 102.76 (quaternary ArC), 88.28 ( $2 \mathrm{C}, \mathrm{ArC}$ ), 86.24 ( $2 \mathrm{C}, \mathrm{ArC}), 78.29$ (5 C, $\mathrm{C}_{5} \mathrm{H}_{5}$ ), $63.19\left(2 \mathrm{C}, \mathrm{CO}_{2} \mathrm{CH}_{2}\right), 57.15$ (quaternary aliph.) $21.54(\mathrm{CMe})$, $20.03(\mathrm{Ar} M e)$, and $13.82\left(2 \mathrm{C}, \mathrm{CH}_{2} \mathrm{Me}\right)$.

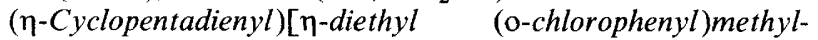
malonate]iron(II) hexafluorophosphate (4d), orange oil $(1.19 \mathrm{~g}$, $72 \%$ ) (Found: $\mathrm{C}, 41.7 ; \mathrm{H}, 4.1 . \mathrm{C}_{19} \mathrm{H}_{22} \mathrm{ClF}_{6} \mathrm{FeO}_{4} \mathrm{P}$ requires $\mathrm{C}$, $41.4 ; \mathrm{H}, 4.0 \%) ; v_{\text {max. }}$ (neat) $1740 \mathrm{~cm}^{-1}(\mathrm{CO}) ; \delta_{\mathrm{H}}\left(\mathrm{CDCl}_{3}\right) 6.62-$ $6.40(3 \mathrm{H}, 2 \mathrm{~m}, \mathrm{ArH}), 6.28(1 \mathrm{H}, \mathrm{br} \mathrm{s}, \mathrm{ArH}), 5.30\left(5 \mathrm{H}, \mathrm{s}, \mathrm{C}_{5} \mathrm{H}_{5}\right)$, $4.46-4.21\left(4 \mathrm{H}, 2 \mathrm{~m}, \mathrm{CO}_{2} \mathrm{CH}_{2}\right), 2.03(3 \mathrm{H}, \mathrm{s}, \mathrm{CMe})$, and 1.42$1.25\left(6 \mathrm{H}, 2 \mathrm{~m}, \mathrm{CO}_{2} \mathrm{CH}_{2} \mathrm{Me}\right) ; \delta_{\mathrm{C}}\left(\mathrm{CD}_{3} \mathrm{CN}\right) 169.73\left(\mathrm{CO}_{2}\right), 168.67$ $\left(\mathrm{CO}_{2}\right), 107.70$ (quaternary $\mathrm{ArC}$ ), 106.35 (quaternary $\mathrm{ArC}$ ), 89.42. 88.93, 87.74, and 87.21 (ArC), $80.56\left(5 \mathrm{C}, \mathrm{C}_{5} \mathrm{H}_{5}\right), 64.16$ $\left(\mathrm{CO}_{2} \mathrm{CH}_{2}\right), 63.95\left(\mathrm{CO}_{2} \mathrm{CH}_{2}\right), 58.57$ (quaternary aliph.), 23.46 (CMe), and $14.05\left(\mathrm{CH}_{2} \mathrm{Me}\right)$.

( $\eta$-Cyclopentadienyl) $[\eta$-diethyl (m-chlorophenyl)methylmalonate]iron(II) hexafluorophosphate $(4 \mathrm{e})$, orange oil $(1.31 \mathrm{~g}$, $79 \%$ ) (Found: C, 41.2; H, 4.0\%); v $v_{\text {max }}$ (neat) $1733 \mathrm{~cm}^{-1}$ (CO); $\delta_{\mathrm{H}}\left(\mathrm{CDCl}_{3}\right) 6.80(1 \mathrm{H}, \mathrm{d}, J 5.7 \mathrm{~Hz}, \mathrm{ArH}), 6.66(1 \mathrm{H}, \mathrm{t}, J 5.7 \mathrm{~Hz}$, ArH $), 6.48(1 \mathrm{H}, \mathrm{s}, \mathrm{ArH}), 6.35(1 \mathrm{H}, \mathrm{d}, J 5.7 \mathrm{~Hz}, \operatorname{ArH}), 5.18(5 \mathrm{H}, \mathrm{s}$, $\left.\mathrm{C}_{5} \mathrm{H}_{5}\right), 4.42-4.31\left(4 \mathrm{H}, \mathrm{m}, \mathrm{CO}_{2} \mathrm{CH}_{2}\right), 1.97(3 \mathrm{H}, \mathrm{s}, \mathrm{CMe})$, and $1.44-1.25\left(6 \mathrm{H}, \mathrm{m}, \mathrm{CH}_{2} \mathrm{Me}\right) ; \delta_{\mathrm{C}}\left(\mathrm{CD}_{3} \mathrm{CN}\right) 169.57\left(\mathrm{CO}_{2}\right), 169.51$ $\left(\mathrm{CO}_{2}\right), 107.63$ (quaternary $\mathrm{ArC}$ ), 105.07 (quaternary $\mathrm{ArC}$ ), 89.22, 87.97, 87.60, and 86.35 (ArC), 80.63 (5 C, $\left.\mathrm{C}_{5} \mathrm{H}_{5}\right), 64.00$ $\left(\mathrm{CO}_{2} \mathrm{CH}_{2}\right), 63.91\left(\mathrm{CO}_{2} \mathrm{CH}_{2}\right), 57.58$ (quaternary aliph.), 21.96 (CMe), and $14.04\left(2 \mathrm{C}, \mathrm{CH}_{2} \mathrm{Me}\right)$.

( $\eta$-Cyclopentadienyl) $[\eta$-diethyl

(p-chlorophenyl)methylmalonate]iron(II) hexafluorophosphate (4f), orange, solidifying oil $(1.26 \mathrm{~g}, 76 \%$ ) (Found: C, 41.7; H, 4.15\%); v max. (neat) 1732 $\mathrm{cm}^{-1}(\mathrm{CO}) ; \delta_{\mathrm{H}}\left(\mathrm{CDCl}_{3}\right) 6.69(2 \mathrm{H}, \mathrm{d}, J 6.6 \mathrm{~Hz}, \mathrm{ArH}), 6.48(2 \mathrm{H}, \mathrm{d}$, $J 6.6 \mathrm{~Hz}, \mathrm{ArH}), 5.18\left(5 \mathrm{H}, \mathrm{s}, \mathrm{C}_{5} \mathrm{H}_{5}\right), 4.38-4.32(4 \mathrm{H}, \mathrm{m}$, $\left.\mathrm{CO}_{2} \mathrm{CH}_{2}\right), 1.94(3 \mathrm{H}, \mathrm{s}, \mathrm{CMe})$, and $1.34(6 \mathrm{H}, \mathrm{t}, J 7.0 \mathrm{~Hz}$, $\left.\mathrm{CH}_{2} \mathrm{Me}\right) ; \delta_{\mathrm{C}}\left(\mathrm{CD}_{3} \mathrm{CN}\right) 169.67\left(2 \mathrm{C}, \mathrm{CO}_{2}\right), 107.73$ (quaternary $\mathrm{ArC}), 104.11$ (quaternary $\mathrm{ArC}), 88.65(2 \mathrm{C}, \mathrm{ArC}), 87.22(2 \mathrm{C}$, $\mathrm{ArC}), 80.77\left(5 \mathrm{C}, \mathrm{C}_{5} \mathrm{H}_{5}\right), 63.99\left(2 \mathrm{C}, \mathrm{CO}_{2} \mathrm{CH}_{2}\right), 57.42$ (quaternary aliph.), 22.11 (CMe), and $14.18\left(2 \mathrm{C}, \mathrm{CH}_{2} \mathrm{Me}\right)$.

$(\eta-C y c l o p e n t a d i e n y l)[\eta$-diethyl (p-chlorophenyl)ethylmalonate]iron(II) hexafluorophosphate (5f), orange-yellow, solidifying oil $(1.26 \mathrm{~g}, 74 \%$ ) (Found: $\mathrm{C}, 42.2 ; \mathrm{H}, \quad 4.0$. $\mathrm{C}_{20} \mathrm{H}_{24} \mathrm{ClF}_{6} \mathrm{FeO}_{4} \mathrm{P}$ requires $\mathrm{C}, 42.5 ; \mathrm{H}, 4.3 \%$ ); $v_{\text {max. }}$ (neat) $1733 \mathrm{~cm}^{-1}(\mathrm{CO}) ; \delta_{\mathrm{H}}\left(\mathrm{CDCl}_{3}\right) 6.75(2 \mathrm{H}, \mathrm{d}, J 6.0 \mathrm{~Hz}, \mathrm{ArH}), 6.46$ $(2 \mathrm{H}, \mathrm{d}, J 6.0 \mathrm{~Hz}, \mathrm{ArH}), 5.20\left(5 \mathrm{H}, \mathrm{s}, \mathrm{C}_{5} \mathrm{H}_{5}\right), 4.40(4 \mathrm{H}, \mathrm{q}, J$ $\left.6.8 \mathrm{~Hz}, \mathrm{CO}_{2} \mathrm{CH}_{2}\right), 2.30\left(2 \mathrm{H}, \mathrm{q}, J 7.1 \mathrm{~Hz}, \mathrm{CH}_{2} \mathrm{Me}\right), 1.38(6 \mathrm{H}, \mathrm{t}, J$ $6.8 \mathrm{~Hz}, \mathrm{CO}_{2} \mathrm{CH}_{2} \mathrm{Me}$ ), and $0.87\left(3 \mathrm{H}, \mathrm{t}, J 7.1 \mathrm{~Hz}, \mathrm{CH}_{2} \mathrm{Me}\right)$; $\delta_{\mathrm{C}}\left(\left[{ }^{2} \mathrm{H}_{6}\right]\right.$ acetone) $167.96\left(2 \mathrm{C}, \mathrm{CO}_{2}\right), 106.63$ (quaternary $\left.\mathrm{ArC}\right)$, 102.73 (quaternary $\mathrm{ArC}), 88.18$ (2 C, $\mathrm{ArC}), 87.10$ (2 C, $\mathrm{ArC}$ ), $80.45\left(5 \mathrm{C}, \mathrm{C}_{5} \mathrm{H}_{5}\right), 63.06\left(2 \mathrm{C}, \mathrm{CO}_{2} \mathrm{CH}_{2}\right), 60.68$ (quaternary aliph.), $30.23\left(\mathrm{CCH}_{2} \mathrm{Me}\right), 13.66\left(2 \mathrm{C}, \mathrm{CO}_{2} \mathrm{CH}_{2} \mathrm{Me}\right)$, and 8.95 $\left(\mathrm{CCH}_{2} \mathrm{Me}\right)$.

$(\eta-C y c l o p e n t a d i e n y l)\left[\eta\right.$-tetraethyl $\alpha, \alpha^{\prime}$-diethyl-m-phenylenedimalonate)iron(II) hexafluorophosphate (8e), yellow crystals
(1.46 g, 68\%) (Found: $\mathrm{C}, 48.4 ; \mathrm{H}, 5.4 . \mathrm{C}_{29} \mathrm{H}_{39} \mathrm{~F}_{6} \mathrm{FeO}_{8} \mathrm{P}$ requires $\mathrm{C}, 48.55 ; \mathrm{H}, 5.5 \%) ; v_{\max }(\mathrm{KBr}) 1733 \mathrm{~cm}^{-1}(\mathrm{CO}) ; \delta_{\mathrm{H}}\left(\mathrm{CDCl}_{3}\right)$ 6.60-6.52 (1 H, m, ArH $), 6.49(1 \mathrm{H}, \mathrm{s}, \mathrm{ArH}), 6.42-6.32(2 \mathrm{H}, \mathrm{m}$, $\mathrm{ArH}), 5.06\left(5 \mathrm{H}, \mathrm{s}, \mathrm{C}_{5} \mathrm{H}_{5}\right), 4.52-4.38\left(8 \mathrm{H}, \mathrm{m}, \mathrm{CO}_{2} \mathrm{CH}_{2}\right), 2.35$ $2.26\left(4 \mathrm{H}, \mathrm{m}, \mathrm{CCH}_{2} \mathrm{Me}\right), 1.44-1.31\left(12 \mathrm{H}, \mathrm{m}, \mathrm{CO}_{2} \mathrm{CH}_{2} \mathrm{Me}\right)$, and $0.91-0.79\left(6 \mathrm{H}, \mathrm{m}, \mathrm{CCH}_{2} \mathrm{Me}\right) ; \delta_{\mathrm{C}}\left(\left[{ }^{2} \mathrm{H}_{6}\right]\right.$ acetone $) 169.89(4 \mathrm{C}$, $\left.\mathrm{CO}_{2}\right), 103.45(2 \mathrm{C}$, quaternary $\mathrm{ArC}), 88.21,87.44$, and $86.63(2 \mathrm{C}$, $\mathrm{ArC}), 79.56\left(5 \mathrm{C}, \mathrm{C}_{5} \mathrm{H}_{5}\right), 63.51\left(2 \mathrm{C}, \mathrm{CO}_{2} \mathrm{CH}_{2}\right), 63.35(2 \mathrm{C}$, $\left.\mathrm{CO}_{2} \mathrm{CH}_{2}\right), 61.84\left(2 \mathrm{C}\right.$, quaternary aliph.), $30.87\left(2 \mathrm{C}, \mathrm{CCH}_{2} \mathrm{Me}\right)$, $14.07\left(4 \mathrm{C}, \mathrm{CO}_{2} \mathrm{CH}_{2} \mathrm{Me}\right.$ ), and $9.42\left(2 \mathrm{C}, \mathrm{CCH}_{2} \mathrm{Me}\right)$.

$(\eta-C y c l o p e n t a d i e n y l)\left(\eta\right.$-tetraethyl $\alpha, \alpha^{\prime}$-diethyl-p-phenylenedimalonate)iron(II) hexafluorophosphate (8f), yellow crystals $\left(1.42 \mathrm{~g}, 66 \%\right.$ ) (Found: $\mathrm{C}, 48.7 ; \mathrm{H}, 5.6 \%$ ); $v_{\max }(\mathrm{KBr}) 1735 \mathrm{~cm}^{-1}$ (CO); $\delta_{\mathrm{H}}\left(\mathrm{CDCl}_{3}\right) 6.42(4 \mathrm{H}$, br s, $\mathrm{ArH}), 5.07\left(5 \mathrm{H}, \mathrm{s}, \mathrm{C}_{5} \mathrm{H}_{5}\right)$, $4.48-4.37\left(8 \mathrm{H}, \mathrm{m}, \mathrm{CO}_{2} \mathrm{CH}_{2}\right), 2.31\left(4 \mathrm{H}, \mathrm{q}, J 7.3 \mathrm{~Hz}, \mathrm{CCH}_{2} \mathrm{Me}\right)$, $1.38\left(12 \mathrm{H}, \mathrm{t}, J 7.1 \mathrm{~Hz}, \mathrm{CO}_{2} \mathrm{CH}_{2} \mathrm{Me}\right)$, and $0.83(6 \mathrm{H}, \mathrm{t}, J 7.3 \mathrm{~Hz}$, $\left.\mathrm{CCH}_{2} \mathrm{Me}\right) ; \delta_{\mathrm{C}}\left(\mathrm{CD}_{3} \mathrm{CN}\right) 169.02\left(4 \mathrm{C}, \mathrm{CO}_{2}\right), 104.04(2 \mathrm{C}$, quaternary $\mathrm{ArC}), 86.46(4 \mathrm{C}, \mathrm{ArC}), 79.34\left(5 \mathrm{C}, \mathrm{C}_{5} \mathrm{H}_{5}\right), 63.54(4 \mathrm{C}$, $\left.\mathrm{CO}_{2} \mathrm{CH}_{2}\right), 61.67\left(2 \mathrm{C}\right.$, quaternary aliph.), $31.26\left(2 \mathrm{C}, \mathrm{CCH}_{2} \mathrm{Me}\right)$, $13.97\left(4 \mathrm{C}, \mathrm{CO}_{2} \mathrm{CH}_{2} \mathrm{Me}\right.$ ), and $9.34\left(2 \mathrm{C}, \mathrm{CCH}_{2} \mathrm{Me}\right)$.

( $\eta$-Cyclopentadienyl $)(\eta-$ diethyl methyl $(\mathrm{m}-$ morpholinophenyl $)-$ malonate]iron(II) Hexafluorophosphate (10).-A solution of the complex (4e) $(2 \mathrm{mmol})$ and morpholine $(5 \mathrm{mmol})$ in methylene dichloride $(10 \mathrm{ml})$ was stirred under nitrogen at room temperature for $5 \mathrm{~h}$. The resulting dark red solution was evaporated to dryness, the residue was redissolved in methylene dichloride $(50 \mathrm{ml})$, and the solution was washed consecutively with dil. hydrochloric acid $(10 \% ; 10 \mathrm{ml})$, aq. ammonium hexafluorophosphate $(2 \mathrm{mmol}$ in $10 \mathrm{ml})$, and water $(3 \times 10 \mathrm{ml})$. The orange solution was then dried over magnesium sulphate and evaporated to dryness, and the residue was washed with diethyl ether $(2 \times 20 \mathrm{ml})$ and dried under reduced pressure, giving the pure product $(10)$ as an orange-brown oil $(0.94 \mathrm{~g}, 78 \%)$ (Found: $\mathrm{C}, 45.7 ; \mathrm{H}, 5.0 ; \mathrm{N}, 2.2 . \mathrm{C}_{23} \mathrm{H}_{30} \mathrm{~F}_{6} \mathrm{FeNO}_{5} \mathrm{P}$ requires $\mathrm{C}, 45.9 ; \mathrm{H}$, $5.0 ; \mathrm{N}, 2.3 \%) ; v_{\max }$. (neat) $1730 \mathrm{~cm}^{-1}(\mathrm{CO}) ; \delta_{\mathrm{H}}\left(\mathrm{CDCl}_{3}\right) 6.23(1 \mathrm{H}$, t, $J 6.6 \mathrm{~Hz}, \mathrm{ArH}), 6.12(1 \mathrm{H}, \mathrm{d}, J 7.0 \mathrm{~Hz}, \mathrm{ArH}), 6.02(1 \mathrm{H}, \mathrm{d}, J 6.2$ $\mathrm{Hz}, \mathrm{ArH}), 5.74(1 \mathrm{H}, \mathrm{s}, \mathrm{ArH}), 4.95\left(5 \mathrm{H}, \mathrm{s}, \mathrm{C}_{5} \mathrm{H}_{5}\right), 4.42-4.28$ $\left(4 \mathrm{H}, \mathrm{m}, \mathrm{CO}_{2} \mathrm{CH}_{2}\right), 3.88\left(4 \mathrm{H}, \mathrm{t}, \mathrm{J} 4.7 \mathrm{~Hz}, \mathrm{CH}_{2} \mathrm{CH}_{2} \mathrm{O}\right), 3.42-$ $3.28\left(4 \mathrm{H}, \mathrm{m}, \mathrm{CH}_{2} \mathrm{~N}\right), 1.95(3 \mathrm{H}, \mathrm{s}, \mathrm{CMe})$, and $1.40-1.32(6 \mathrm{H}, \mathrm{m}$, $\left.\mathrm{CH}_{2} \mathrm{Me}\right) ; \delta_{\mathrm{C}}\left(\left[{ }^{2} \mathrm{H}_{6}\right]\right.$ acetone $) 169.89\left(\mathrm{CO}_{2}\right), 169.43\left(\mathrm{CO}_{2}\right), 126.10$ (quaternary $\mathrm{ArC}$ ), 102.27 (quaternary $\mathrm{ArC}$ ), 85.59, 81.15, and 79.03 (ArC), 76.37 (5 C, $\left.\mathrm{C}_{5} \mathrm{H}_{5}\right), 68.90$ (ArC), 66.25 (2 C, $\left.\mathrm{CH}_{2} \mathrm{CH}_{2} \mathrm{O}\right), \quad 63.24 \quad\left(\mathrm{CO}_{2} \mathrm{CH}_{2}\right), \quad 63.14 \quad\left(\mathrm{CO}_{2} \mathrm{CH}_{2}\right), \quad 57.67$ (quaternary aliph.), $46.99\left(2 \mathrm{C}, \mathrm{CH}_{2} \mathrm{~N}\right), 21.59(\mathrm{CMe})$, and 14.03 $\left(2 \mathrm{C}, \mathrm{CH}_{2} \mathrm{Me}\right)$.

$\{1-5-\eta-1-C h l o r o-6-e x o-c y a n o-2-[1,1-b i s($ ethoxycarbonyl)ethyl $]$ cyclohexadienyl $\}$ ( $\eta$-cyclopentadienyl)iron(II) (12a) and $\{1-5-\eta$-2-Chloro-6-exo-cyano-3-[1,1-bis(ethoxycarbonyl)-

ethyl $]$ cyclohexadienyl $\}$ ( $\eta$-cyclopentadienyl)iron(II) (12b).-To a stirred solution of complex (4d) $(0.55 \mathrm{~g}, 1 \mathrm{mmol})$ in DMF $(5 \mathrm{ml})$ was added powdered $\mathrm{NaCN}(0.3 \mathrm{~g}, 6.12 \mathrm{mmol})$ followed by water ( 1 drop). After the mixture had been stirred vigorously for $30 \mathrm{~min}$ the products were extracted with diethyl ether $(3 \times 50 \mathrm{ml})$. The combined extract was washed with water $(3 \times 30 \mathrm{ml})$, dried over magnesium sulphate, and then evaporated to dryness. The resulting products were purified by passage through a short $(5 \mathrm{~cm})$ column filled with Alumina F20 with carbon tetrachloride to elute any impurities and then with chloroform to elute the products. Upon evaporation of the dried (magnesium sulphate) chloroform solution, a mixture of the products was obtained as a yellow-brown oil $(0.38 \mathrm{~g}, 88 \%)$ (Found: $\mathrm{C}, 56.1 ; \mathrm{H}, 5.15 ; \mathrm{N}, 3.4 . \mathrm{C}_{20} \mathrm{H}_{22} \mathrm{ClFeNO}_{4}$ requires $\mathrm{C}$, $55.7 ; \mathrm{H}, 5.1 ; \mathrm{N}, 3.2 \%$ ); $v_{\text {max. }}$ (neat) 1740 and $1730(\mathrm{CO})$ and $2210 \mathrm{~cm}^{-1}(\mathrm{CN}) ; \delta_{\mathrm{H}}(\mathbf{1 2 a})\left(\mathrm{CDCl}_{3}\right) 5.97(1 \mathrm{H}, 2 \mathrm{~d}, J 5.5$ and 1.3 $\mathrm{Hz}), 4.58\left(5 \mathrm{H}, \mathrm{s}, \mathrm{C}_{5} \mathrm{H}_{5}\right), 4.49(1 \mathrm{H}, 3 \mathrm{~d}, J 6.1,5.5$ and $0.5 \mathrm{~Hz})$, $4.40-4.20\left(4 \mathrm{H}, \mathrm{m}, \mathrm{CO}_{2} \mathrm{CH}_{2}\right), 3.85(1 \mathrm{H}, 2 \mathrm{~d}, J 6.8$ and $0.5 \mathrm{~Hz})$, 
$3.06(1 \mathrm{H}, 3 \mathrm{~d}, J 6.8,6.1$, and $1.3 \mathrm{~Hz}), 1.88(3 \mathrm{H}, \mathrm{s}, \mathrm{CMe})$, and $1.36-1.20\left(6 \mathrm{H}, \mathrm{m}, \mathrm{CO}_{2} \mathrm{CH}_{2} \mathrm{Me}\right) ; \delta_{\mathrm{H}}(\mathbf{1 2 b})\left(\mathrm{CDCl}_{3}\right) 4.52(5 \mathrm{H}, \mathrm{s}$, $\left.\mathrm{C}_{5} \mathrm{H}_{5}\right), 4.40-4.20\left(4 \mathrm{H}, \mathrm{m}, \mathrm{CO}_{2} \mathrm{CH}_{2}+1 \mathrm{H}, \mathrm{ArH}\right), 3.55(1 \mathrm{H}, \mathrm{t}$, $J 6.5$ and $\mathrm{d}, J 0.7 \mathrm{~Hz}), 2.82(1 \mathrm{H}, 2 \mathrm{~d}, J 6.5$ and $1.5 \mathrm{~Hz}), 2.47(1 \mathrm{H}$, $\mathrm{t}, J 6.5$ and d, $J 1.5 \mathrm{~Hz}), 2.24(3 \mathrm{H}, \mathrm{s}, \mathrm{CMe})$, and $1.63-1.20(6 \mathrm{H}$, $\left.\mathrm{m}, \mathrm{CO}_{2} \mathrm{CH}_{2} \mathrm{Me}\right) ; \delta_{\mathrm{C}}(\mathbf{1 2 a})\left(\mathrm{CDCl}_{3}\right) 170.33\left(\mathrm{CO}_{2}\right), 170.25\left(\mathrm{CO}_{2}\right)$, $115.84(\mathrm{CN}), 94.46$ (quaternary $\mathrm{ArC}, \mathrm{C}-2), 78.70(\mathrm{C}-3), 77.76$ $\left(5 \mathrm{C}, \mathrm{C}_{5} \mathrm{H}_{5}\right), 75.85(\mathrm{C}-4), 61.99\left(\mathrm{CO}_{2} \mathrm{CH}_{2}\right), 61.88\left(\mathrm{CO}_{2} \mathrm{CH}_{2}\right)$, 57.55 (quaternary aliph.), 50.77 (quaternary $\mathrm{ArC}, \mathrm{C}-1), 38.95$ (C-6), $27.22(\mathrm{C}-5), 24.12(\mathrm{CMe}), 13.72\left(\mathrm{CO}_{2} \mathrm{CH}_{2} \mathrm{Me}\right)$, and 13.67 $\left(\mathrm{CO}_{2} \mathrm{CH}_{2} \mathrm{Me}\right) ; \delta_{\mathrm{C}}(\mathbf{1 2 b})\left(\mathrm{CDCl}_{3}\right) 170.51\left(\mathrm{CO}_{2}\right), 170.13\left(\mathrm{CO}_{2}\right)$, $117.59(\mathrm{CN}), 102.57$ (quaternary $\mathrm{ArC}, \mathrm{C}-2), 99.75$ (quaternary $\mathrm{ArC}, \mathrm{C}-3), 80.08$ (C-4), $77.09\left(5 \mathrm{C}, \mathrm{C}_{5} \mathrm{H}_{5}\right), 62.09\left(\mathrm{CO}_{2} \mathrm{CH}_{2}\right)$, $61.83\left(\mathrm{CO}_{2} \mathrm{CH}_{2}\right), 57.13$ (quaternary aliph.), 27.37 (C-6), 24.46 (CMe), 21.80 (C-1), 20.23 (C-5), and $13.63\left(2 \mathrm{C}, \mathrm{CO}_{2} \mathrm{CH}_{2} \mathrm{Me}\right)$. Ratio of the isomers (12a): (12b) calculated from the integration of signals in the ${ }^{1} \mathrm{H}$ n.m.r. spectrum and in the inverse gated ${ }^{13} \mathrm{C}$ spectrum (pulse delay 30 s) was $\sim 6: 1$.

Decomplexation and Liberation of Diethyl Alkyl(phenyl)malonates.-Cationic products (4), (5), and (10) were demetallated by the pyrolytic sublimation process described previously ${ }^{14}$ Diethyl alkyl(phenyl)malonates were purified on a short column of silica gel (twice if necessary) with heptane and carbon tetrachloride for washing and chloroform for the elution of the product.

Compounds (13a and $\mathbf{b})$ were liberated from their respective complexes (12a and b) by a procedure employing DDQ as described recently ${ }^{22}$ and were purified as described above.

Diethyl ethyl(phenyl)malonate (7a) was an oil ${ }^{23}(78 \%)$, (Found: $\mathrm{C}, 68.4 ; \mathrm{H}, 7.85$. Calc. for $\mathrm{C}_{15} \mathrm{H}_{20} \mathrm{O}_{4}: \mathrm{C}, 68.2 ; \mathrm{H}, 7.6 \%$ ); $v_{\max }$ (neat) $1730 \mathrm{~cm}^{-1} ; m / z 264\left(M^{+}, 4.4 \%\right), 119(68)$, and 91 $(100) ; \delta_{\mathrm{H}}\left(\mathrm{CDCl}_{3}\right) 7.45-7.27(5 \mathrm{H}$, multiplets, ArH), 4.27--4.15 $\left(4 \mathrm{H}, \mathrm{m}, \mathrm{CO}_{2} \mathrm{CH}_{2}\right), 2.35\left(2 \mathrm{H}, \mathrm{q}, J 7.4 \mathrm{~Hz}, \mathrm{CCH}_{2} \mathrm{Me}\right), 1.23(6 \mathrm{H}, \mathrm{t}$, $J 7.1 \mathrm{~Hz}, \mathrm{CO}_{2} \mathrm{CH}_{2} \mathrm{Me}$ ), and $0.88\left(3 \mathrm{H}, \mathrm{t}, J 7.4 \mathrm{~Hz}, \mathrm{CCH}_{2} \mathrm{Me}\right)$; $\delta_{\mathrm{C}}\left(\mathrm{CDCl}_{3}\right) 170.56\left(2 \mathrm{C}, \mathrm{CO}_{2}\right), 136.79$ (quaternary $\mathrm{ArC}$ ), 127.97 (2 C, $\mathrm{ArC}), 127.90(2 \mathrm{C}, \mathrm{ArC}$ ), 127.22 (ArC), 62.94 (quaternary aliph.), $61.22\left(2 \mathrm{C}, \mathrm{CO}_{2} \mathrm{CH}_{2}\right), 28.73\left(\mathrm{CCH}_{2} \mathrm{Me}\right), 13.84(2 \mathrm{C}$, $\left.\mathrm{CO}_{2} \mathrm{CH}_{2} \mathrm{Me}\right)$, and $9.15\left(\mathrm{CCH}_{2} \mathrm{Me}\right)$.

Diethyl ethyl(o-tolyl)malonate (7b) was an oil ( $73 \%$ ), (Found: $\mathrm{C}, 69.3 ; \mathrm{H}, 7.8 . \mathrm{C}_{16} \mathrm{H}_{22} \mathrm{O}_{4}$ requires $\mathrm{C}, 69.0 ; \mathrm{H}, 8.0 \%$ ); $v_{\max }$ (neat) $1735 \mathrm{~cm}^{-1}(\mathrm{CO}) ; m / z 278\left(M^{+}, 6.9 \%\right), 232$ (72.2), and $204(100)$; $\delta_{\mathrm{H}}\left(\mathrm{CDCl}_{3}\right) 7.21-7.12(4 \mathrm{H}, \mathrm{m}, \mathrm{ArH}), 4.23$ and $4.22(4 \mathrm{H}, 2 \mathrm{q}, J$ $\left.7.1 \mathrm{~Hz}, \mathrm{CO}_{2} \mathrm{CH}_{2}\right), 2.40\left(2 \mathrm{H}, \mathrm{q}, J 7.4 \mathrm{~Hz}, \mathrm{CCH}_{2} \mathrm{Me}\right), 2.28(3 \mathrm{H}$, $\mathrm{s}, \mathrm{ArMe}), 1.24\left(6 \mathrm{H}, \mathrm{t}, J 7.1 \mathrm{~Hz}, \mathrm{CO}_{2} \mathrm{CH}_{2} \mathrm{Me}\right)$, and $0.91(3 \mathrm{H}, \mathrm{t}$, $\left.J 7.4 \mathrm{~Hz}, \mathrm{CCH}_{2} \mathrm{Me}\right) ; \delta_{\mathrm{C}}\left(\mathrm{CDCl}_{3}\right) 170.86\left(2 \mathrm{C}, \mathrm{CO}_{2}\right), 137.19$ (quaternary ArC), 136.21 (quaternary ArC), 131.93, 127.76, 127.25, and 125.58 (ArC), 63.02 (quaternary aliph.), $61.36(2 \mathrm{C}$, $\left.\mathrm{CO}_{2} \mathrm{CH}_{2}\right), 28.39\left(\mathrm{CCH}_{2} \mathrm{Me}\right), 20.97$ (ArMe), 13.87 (2 C, $\left.\mathrm{CO}_{2} \mathrm{CH}_{2} \mathrm{Me}\right)$, and $9.91\left(\mathrm{CCH}_{2} \mathrm{Me}\right)$.

Diethyl methyl(p-tolyl)malonate (6c) was an oil $(72 \%)$, (Found: $\mathrm{C}$, 68.55; $\mathrm{H}, 7.7 . \mathrm{C}_{15} \mathrm{H}_{20} \mathrm{O}_{4}$ requires $\mathrm{C}, 68.2 ; \mathrm{H}, 7.6 \%$ ); $v_{\text {max. }}$ (neat) $1735 \mathrm{~cm}^{-1}$ (CO); $m / z 264\left(M^{+}, 41.1 \%\right.$ ) and $191(100)$; $\delta_{\mathrm{H}}\left(\mathrm{CDCl}_{3}\right) 7.26(2 \mathrm{H}, \mathrm{d}, J 8.3 \mathrm{~Hz}, \mathrm{ArH}), 7.15(2 \mathrm{H}, \mathrm{d}, J 8.3 \mathrm{~Hz}$, $\mathrm{ArH}), 4.23$ and $4.22\left(4 \mathrm{H}, 2 \mathrm{q}, J 7.1 \mathrm{~Hz}, \mathrm{CO}_{2} \mathrm{CH}_{2}\right), 2.33(3 \mathrm{H}, \mathrm{s}$, $\mathrm{ArMe}), 1.84(3 \mathrm{H}, \mathrm{s}, \mathrm{CMe})$, and $1.25(6 \mathrm{H}, \mathrm{t}, J 7.1 \mathrm{~Hz}$, $\mathrm{CO}_{2} \mathrm{CH}_{2} \mathrm{Me}$ ); $\delta_{\mathrm{C}}\left(\mathrm{CDCl}_{3}\right) 171.50\left(2 \mathrm{C}, \mathrm{CO}_{2}\right), 137.06$ (quaternary $\mathrm{ArC}), 135.23$ (quaternary $\mathrm{ArC}$ ), 128.69 (2 C, ArC), 127.15 (2 C, ArC), 61.44 ( $2 \mathrm{C}, \mathrm{CO}_{2} \mathrm{CH}_{2}$ ), 58.28 (quaternary aliph.), 22.15 $(\mathrm{CMe}), 20.82(\mathrm{ArMe})$, and $13.82\left(2 \mathrm{C}, \mathrm{CO}_{2} \mathrm{CH}_{2} \mathrm{Me}\right)$.

Diethyl o-chlorophenyl(methyl)malonate (6d) was an oil (72\%) (Found: $\mathrm{C}, 59.4 ; \mathrm{H}, 6.2 ; \mathrm{C}_{14} \mathrm{H}_{17} \mathrm{ClO}_{4}$ requires $\mathrm{C}, 59.1 ; \mathrm{H}$, $6.0 \%$; $v_{\text {max }}$ (neat) $1740 \mathrm{~cm}^{-1}(\mathrm{CO}) ; \mathrm{m} / z 286\left[M^{+}\left({ }^{37} \mathrm{Cl}\right), 0.2 \%\right]$, $284\left[M^{+}\left({ }^{35} \mathrm{Cl}\right), 0.6\right]$, and $249(100) ; \delta_{\mathrm{H}}\left(\mathrm{CDCl}_{3}\right) 7.40-7.36(1 \mathrm{H}$, $\mathrm{m}, \mathrm{ArH}), 7.25-7.22(2 \mathrm{H}, \mathrm{m}, \mathrm{ArH}), 7.18-7.15(1 \mathrm{H}, \mathrm{m}, \mathrm{ArH})$, $4.38-4.18\left(4 \mathrm{H}, \mathrm{m}, \mathrm{CO}_{2} \mathrm{CH}_{2}\right), 1.91(3 \mathrm{H}, \mathrm{s}, \mathrm{CMe})$, and 1.27 $\left(6 \mathrm{H}, \mathrm{t}, J 7.1 \mathrm{~Hz}, \mathrm{CO}_{2} \mathrm{CH}_{2} \mathrm{Me}\right) ; \delta_{\mathrm{C}}\left(\mathrm{CDCl}_{3}\right) 170.22\left(2 \mathrm{C}, \mathrm{CO}_{2}\right)$, 137.45 (quaternary $\mathrm{ArC}$ ), 133.44 (quaternary ArC), 130.78,
128.43, 128.04, and 126.64 ( $\mathrm{ArC}), 61.71\left(2 \mathrm{C}, \mathrm{CO}_{2} \mathrm{CH}_{2}\right), 59.71$ (quaternary aliph.), $21.49(\mathrm{CMe})$, and $13.60\left(2 \mathrm{C}, \mathrm{CO}_{2} \mathrm{CH}_{2} \mathrm{Me}\right)$. Diethyl m-chlorophenyl(methyl)malonate (6e) was an oil (76\%) (Found: C, 59.45; H, 6.1\%); $v_{\max .}$ (neat) $1735 \mathrm{~cm}^{-1}$ (CO); $m / z 286\left[M^{+}\left({ }^{37} \mathrm{Cl}\right), 0.9 \%\right], 284\left[M^{+}\left({ }^{35} \mathrm{Cl}\right), 2.4\right], 211(9.1)$, and $186(100) ; \delta_{\mathrm{H}}\left(\mathrm{CDCl}_{3}\right) 7.38(1 \mathrm{H}$, br s, $\mathrm{ArH}), 7.32-7.26(3 \mathrm{H}, \mathrm{m}$, $\mathrm{ArH}), 4.23\left(4 \mathrm{H}, \mathrm{q}, J 7.1 \mathrm{~Hz}, \mathrm{CO}_{2} \mathrm{CH}_{2}\right), 1.85(3 \mathrm{H}, \mathrm{s}, \mathrm{CMe})$, and $1.26\left(6 \mathrm{H}, \mathrm{t}, J 7.1 \mathrm{~Hz}, \mathrm{CO}_{2} \mathrm{CH}_{2} \mathrm{Me}\right) ; \delta_{\mathrm{C}}\left(\mathrm{CDCl}_{3}\right) 170.53(2 \mathrm{C}$, $\mathrm{CO}_{2}$ ), 140.01 (quaternary $\mathrm{ArC}$ ), 133.66 (quaternary $\mathrm{ArC}$ ), $129.06,127.52,127.45$, and $125.56(\mathrm{ArC}), 61.53\left(2 \mathrm{C}, \mathrm{CO}_{2} \mathrm{CH}_{2}\right)$, 58.18 (quaternary aliph.), $21.90(\mathrm{CMe})$ and $13.62(2 \mathrm{C}$, $\mathrm{CO}_{2} \mathrm{CH}_{2} \mathrm{Me}$ ).

Diethyl p-chlorophenyl(methyl)malonate (6f) was an oil ( $80 \%)$ (Found: C, 59.3; H, 6.3\%); $v_{\max }$ (neat) $1732 \mathrm{~cm}^{-1}(\mathrm{CO}) ; \mathrm{m} / z 286$ $\left[M^{+}\left({ }^{37} \mathrm{Cl}\right), 10.4^{\%} \%\right], 284\left[M^{+}\left({ }^{35} \mathrm{Cl}\right), 29.8\right], 213(39.7)$, and 211 $(100) ; \delta_{\mathrm{H}}\left(\mathrm{CDCl}_{3}\right) 7.32(4 \mathrm{H}$, br s, ArH $), 4.25(4 \mathrm{H}, \mathrm{q}, J 7.0 \mathrm{~Hz}$, $\left.\mathrm{CO}_{2} \mathrm{CH}_{2}\right), 1.84(3 \mathrm{H}, \mathrm{s}, \mathrm{CMe})$, and $1.25(6 \mathrm{H}, \mathrm{t}, J 7.0 \mathrm{~Hz}$, $\left.\mathrm{CO}_{2} \mathrm{CH}_{2} \mathrm{Me}\right) ; \delta_{\mathrm{C}}\left(\mathrm{CDCl}_{3}\right) 171.11\left(2 \mathrm{C}, \mathrm{CO}_{2}\right), 136.70$ (quaternary $\mathrm{ArC}$ ), 133.56 (quaternary $\mathrm{ArC}), 128.93$ (2 C, $\mathrm{ArC}), 128.22$ (2 C, $\mathrm{ArC}$ ), 61.80 ( $2 \mathrm{C}, \mathrm{CO}_{2} \mathrm{CH}_{2}$ ), 58.22 (quaternary aliph.), 22.10 $(\mathrm{CMe})$, and $13.90\left(2 \mathrm{C}, \mathrm{CO}_{2} \mathrm{CH}_{2} \mathrm{Me}\right)$.

Diethyl p-chlorophenyl(ethyl)malonate $(7 \mathrm{f})$ was an oil ${ }^{7}(78 \%)$ (Found C, 60.0; H, 6.2. Calc. for $\mathrm{C}_{15} \mathrm{H}_{19} \mathrm{ClO}_{4}: \mathrm{C}, 60.3 ; \mathrm{H}, 6.4 \%$ ); $v_{\max }$ (neat) $1735 \mathrm{~cm}^{-1}(\mathrm{CO}) ; m / z 300\left[M^{+}\left({ }^{37} \mathrm{Cl}\right), 0.3 \%\right], 298$ $\left[M^{+}\left({ }^{35} \mathrm{Cl}\right), 0.8\right]$, and $186(100) ; \delta_{\mathrm{H}}\left(\mathrm{CDCl}_{3}\right) 7.39(2 \mathrm{H}, \mathrm{d}, J$ $8.7 \mathrm{~Hz}, \mathrm{ArH}), 7.30(2 \mathrm{H}, \mathrm{d}, J 8.7 \mathrm{~Hz}, \mathrm{ArH}), 4.26-4.17(4 \mathrm{H}, \mathrm{m}$, $\left.\mathrm{CO}_{2} \mathrm{CH}_{2}\right), 2.33\left(2 \mathrm{H}, \mathrm{q}, J 7.4 \mathrm{~Hz}, \mathrm{CCH}_{2} \mathrm{Me}\right), 1.23(6 \mathrm{H}, \mathrm{t}, J 7.1$ $\mathrm{Hz}, \mathrm{CO}_{2} \mathrm{CH}_{2} \mathrm{Me}$ ), and $0.86\left(3 \mathrm{H}, \mathrm{t}, \mathrm{J} 7.4 \mathrm{~Hz}, \mathrm{CCH}_{2} \mathrm{Me}\right)$; $\delta_{\mathrm{C}}\left(\mathrm{CDCl}_{3}\right) 170.12\left(2 \mathrm{C}, \mathrm{CO}_{2}\right), 135.16$ (quaternary $\left.\mathrm{ArC}\right), 133.17$ (quaternary ArC), 129.48 (2 C, ArC), 128.00 (2 C, ArC), 62.34 (quaternary aliph.), $61.33\left(2 \mathrm{C}, \mathrm{CO}_{2} \mathrm{CH}_{2}\right), 28.54\left(\mathrm{CCH}_{2} \mathrm{Me}\right)$, $13.77\left(2 \mathrm{C}, \mathrm{CO}_{2} \mathrm{CH}_{2} \mathrm{Me}\right)$, and $8.95\left(\mathrm{CCH}_{2} \mathrm{Me}\right)$.

Tetraethyl $\alpha, \alpha^{\prime}$-diethyl-m-phenylenedimalonate $(9 \mathrm{e})$ was obtained as a yellowish-white oil $(77 \%$ ) (Found: $\mathrm{C}, 64.4 ; \mathrm{H}, 7.8$. $\mathrm{C}_{24} \mathrm{H}_{34} \mathrm{O}_{8}$ requires $\mathrm{C}, 64.0 ; \mathrm{H}, 7.6 \%$; $v_{\text {max. }}$ (neat) $1735 \mathrm{~cm}^{-1}$ (CO); $m / z 450\left(M^{+}, 2.5 \%\right), 406(4.2), 377$ (13.8), 332 (51.5), 303 (24.4), 135 (31.5), 117 (35.1), 91 (32.9), 83 (33.2), and 73 (100); $\delta_{\mathrm{H}}\left(\mathrm{CDCl}_{3}\right) 7.47(1 \mathrm{H}, \mathrm{br} \mathrm{s}, \mathrm{ArH}), 7.38-7.28(3 \mathrm{H}, \mathrm{m}, \mathrm{ArH})$, $4.29-4.12\left(8 \mathrm{H}, \mathrm{m}, \mathrm{CO}_{2} \mathrm{CH}_{2}\right), 2.33\left(4 \mathrm{H}, \mathrm{q}, J 7.4 \mathrm{~Hz}, \mathrm{CCH}_{2} \mathrm{Me}\right)$, $1.23\left(12 \mathrm{H}, \mathrm{t}, J 7.1 \mathrm{~Hz}, \mathrm{CO}_{2} \mathrm{CH}_{2} \mathrm{Me}\right)$, and $0.87(6 \mathrm{H}, \mathrm{t}, J$ $\left.7.4 \mathrm{~Hz}, \mathrm{CCH}_{2} \mathrm{Me}\right) ; \delta_{\mathrm{C}}\left(\mathrm{CDCl}_{3}\right) 170.11\left(4 \mathrm{C}, \mathrm{CO}_{2}\right), 136.21(2 \mathrm{C}$, quaternary ArC), 128.15 and 127.24 ( $\mathrm{ArC}), 126.75$ ( $2 \mathrm{C}, \mathrm{ArC}$ ), 62.71 (2 C, quaternary aliph.), $60.94\left(4 \mathrm{C}, \mathrm{CO}_{2} \mathrm{CH}_{2}\right), 28.60(2 \mathrm{C}$, $\mathrm{CCH}_{2} \mathrm{Me}$ ), 13.57 (4 C, $\left.\mathrm{CO}_{2} \mathrm{CH}_{2} \mathrm{Me}\right)$, and $8.82\left(2 \mathrm{C}, \mathrm{CCH}_{2} \mathrm{Me}\right.$ ).

Tetraethyl $\alpha, \alpha^{\prime}$-diethyl-p-phenylenedimalonate (9f) was a white solid $\left(80 \%\right.$ ) (Found: C, 64.2; H, 7.5\%); m.p. $58^{\circ} \mathrm{C}$; $v_{\text {max }}{ }^{-}$ (KBr) $1735 \mathrm{~cm}^{-1}(\mathrm{CO}) ; m / z 450\left(M^{+}, 14.6 \%\right), 377(43.8), 304$ $(87.5)$, and $73(100) ; \delta_{\mathrm{H}}\left(\mathrm{CDCl}_{3}\right) 7.39(4 \mathrm{H}$, br s, ArH $), 4.26-4.16$ $\left(8 \mathrm{H}, \mathrm{m}, \mathrm{CO}_{2} \mathrm{CH}_{2}\right), 2.33\left(4 \mathrm{H}, \mathrm{q}, J 7.5 \mathrm{~Hz}, \mathrm{CCH}_{2} \mathrm{Me}\right), 1.23(12 \mathrm{H}$, $\left.\mathrm{t}, J 7.1 \mathrm{~Hz}, \mathrm{CO}_{2} \mathrm{CH}_{2} \mathrm{Me}\right)$, and $0.87\left(6 \mathrm{H}, \mathrm{t}, J 7.5 \mathrm{~Hz}, \mathrm{CCH}_{2} \mathrm{Me}\right)$; $\delta_{\mathrm{C}}\left(\mathrm{CDCl}_{3}\right) 170.39\left(4 \mathrm{C}, \mathrm{CO}_{2}\right), 135.73(2 \mathrm{C}$, quaternary $\mathrm{ArC})$, $127.64(4 \mathrm{C}, \mathrm{ArC}$ ), 62.58 (2 C, quaternary aliph.), 61.19 (4 C, $\left.\mathrm{CO}_{2} \mathrm{CH}_{2}\right), 28.66\left(2 \mathrm{C}, \mathrm{CCH}_{2} \mathrm{Me}\right), 13.77\left(4 \mathrm{C}, \mathrm{CO}_{2} \mathrm{CH}_{2} \mathrm{Me}\right)$, and $9.07\left(2 \mathrm{C}, \mathrm{CCH}_{2} \mathrm{Me}\right.$ ).

Diethyl methyl(m-morpholinophenyl)malonate (11) was an oil (70\%) (Found: $\mathrm{C}, 64.2 ; \mathrm{H}, 7.3 ; \mathrm{N}, 4.3 . \mathrm{C}_{18} \mathrm{H}_{25} \mathrm{NO}_{5}$ requires $\mathrm{C}$, $64.5 ; \mathrm{H}, 7.5 ; \mathrm{N}, 4.2 \%) ; v_{\max }$ (neat) $1735 \mathrm{~cm}^{-1}$ (CO); $\mathrm{m} / z 335\left(\mathrm{M}^{+}\right.$, $2.7 \%$ ) and $186(100) ; \delta_{\mathrm{H}}$ (carbon tetrachloride) $7.11(1 \mathrm{H}, \mathrm{t}, J 8.0$ $\mathrm{Hz}, \mathrm{ArH}), 6.83$ (1 H, br s, ArH), 6.75-6.70 (2 H, m, ArH), 4.16 $\left(4 \mathrm{H}, \mathrm{q}, J 7.0 \mathrm{~Hz}, \mathrm{CO}_{2} \mathrm{CH}_{2}\right), 3.80-3.76\left(4 \mathrm{H}, \mathrm{m}, \mathrm{CH}_{2} \mathrm{CH}_{2} \mathrm{O}\right)$, $3.09-3.06\left(4 \mathrm{H}, \mathrm{m}, \mathrm{CH}_{2} \mathrm{~N}\right), 1.75(3 \mathrm{H}, \mathrm{s}, \mathrm{CMe})$, and $1.24(6 \mathrm{H}, \mathrm{t}$, $\left.J 7.0 \mathrm{~Hz}, \mathrm{CO}_{2} \mathrm{CH}_{2} \mathrm{Me}\right) ; \delta_{\mathrm{C}}\left(\mathrm{CDCl}_{3}\right) 171.30\left(2 \mathrm{C}, \mathrm{CO}_{2}\right), 150.91$ (quaternary $\mathrm{ArC}$ ), 139.10 (quaternary $\mathrm{ArC}$ ), 128.63, 118.91, 115.18, and 114.84 (ArC), $66.70\left(2 \mathrm{C}, \mathrm{CH}_{2} \mathrm{CH}_{2} \mathrm{O}\right), 61.42(2 \mathrm{C}$, $\mathrm{CO}_{2} \mathrm{CH}_{2}$ ), 58.59 (quaternary aliph.), $49.26\left(2 \mathrm{C}, \mathrm{CH}_{2} \mathrm{~N}\right), 22.41$ $\left(\mathrm{CMe}\right.$ ), and $13.83\left(2 \mathrm{C}, \mathrm{CO}_{2} \mathrm{CH}_{2} \mathrm{Me}\right.$ ).

Diethyl 2-chloro-3-cyanophenyl(methyl)malonate (13a) and diethyl 2-chloro-4-cyanophenyl(methyl)malonate (13b) were a 
yellowish oily mixture $(78 \%$ ) (Found: $\mathrm{C}, 58.4 ; \mathrm{H}, 5.5 ; \mathrm{N}, 4.3$. $\mathrm{C}_{15} \mathrm{H}_{16} \mathrm{ClNO}_{4}$ requires $\mathrm{C}, 58.2 ; \mathrm{H}, 5.2 ; \mathrm{N}, 4.5 \%$; $v_{\max .}$ (neat) 1750 and $1735(\mathrm{CO})$ and $2220 \mathrm{~cm}^{-1}(\mathrm{CN}) ; \mathrm{m} / z$ (chemical ionization, methane) $312\left[\bar{M}^{+}\left({ }^{37} \mathrm{Cl}\right), 11 \%\right], 310\left[M^{+}\left({ }^{35} \mathrm{Cl}\right)\right.$, $33.9], 274(30.1), 249(66.1), 213\left[\left({ }^{37} \mathrm{Cl}\right), 40.2\right]$, and $211\left[\left({ }^{35} \mathrm{Cl}\right)\right.$, $100] ; \delta_{\mathrm{H}}\left(\mathrm{CCl}_{4}\right)(13 \mathrm{a}) 7.60(1 \mathrm{H}, 2 \mathrm{~d}, J 7.1$ and $1.1 \mathrm{~Hz}, \mathrm{ArH}), 7.39-$ $7.29(2 \mathrm{H}, \mathrm{m}, \mathrm{ArH}), 4.30-4.14\left(4 \mathrm{H}, \mathrm{m}, \mathrm{CO}_{2} \mathrm{CH}_{2}\right), 1.85(3 \mathrm{H}, \mathrm{s}$, $\mathrm{CMe})$, and $1.29\left(6 \mathrm{H}, \mathrm{t}, J 7.0 \mathrm{~Hz}, \mathrm{CO}_{2} \mathrm{CH}_{2} \mathrm{Me}\right)$; (13b) $7.20-7.15$ $(2 \mathrm{H}, \mathrm{m}, \mathrm{ArH}), 7.08(1 \mathrm{H}, 2 \mathrm{~d}, J 6.8$ and $1.5 \mathrm{~Hz}, \mathrm{ArH}), 4.30-4.14$ (4 $\mathrm{H}$, overlapping, $\left.\mathrm{CO}_{2} \mathrm{CH}_{2}\right), 1.82(3 \mathrm{H}, \mathrm{s}, \mathrm{CMe})$, and $1.27(6 \mathrm{H}$, $\left.\mathrm{t}, J 7.0 \mathrm{~Hz}, \mathrm{CO}_{2} \mathrm{CH}_{2} \mathrm{Me}\right) ; \delta_{\mathrm{C}}\left(\mathrm{CCl}_{4}\right)(13 \mathrm{a}) 168.50\left(2 \mathrm{C}, \mathrm{CO}_{2}\right)$, 139.62 (quaternary $\mathrm{ArC}$ ), 136.10 (quaternary $\mathrm{ArC}$ ), 132.48, 131.90, and $126.68(\mathrm{ArC}), 116.03(\mathrm{CN}), 114.75$ (quaternary ArC), 61.54 ( $2 \mathrm{C}, \mathrm{CO}_{2} \mathrm{CH}_{2}$ ), 59.57 (quaternary aliph.), 21.42 $(\mathrm{CMe})$, and $13.78\left(2 \mathrm{C}, \mathrm{CO}_{2} \mathrm{CH}_{2} \mathrm{Me}\right.$ ); (13b) $169.21\left(2 \mathrm{C}, \mathrm{CO}_{2}\right)$, 142.64 (quaternary $\mathrm{ArC}$ ), 133.49 (quaternary $\mathrm{ArC}$ ), 130.70, 128.18, and $126.35(\mathrm{ArC}), 115.90(\mathrm{CN}), 113.23$ (quaternary $\mathrm{ArC}$ ), 61.04 ( $2 \mathrm{C}, \mathrm{CO}_{2} \mathrm{CH}_{2}$ ), 59.55 (quaternary aliph.), 21.42 $(\mathrm{CMe})$, and $13.72\left(2 \mathrm{C}, \mathrm{CO}_{2} \mathrm{CH}_{2} \mathrm{Me}\right)$. A ratio of isomers (13a):(13b) $4: 1$ was calculated from the integration of the methyl signals in the ${ }^{1} \mathrm{H}$ spectrum and was confirmed from integration of the signals in the inverse gated ${ }^{13} \mathrm{C}$ spectrum.

\section{Acknowledgements}

Financial support given by the Natural Sciences and Engineering Research Council of Canada is gratefully acknowledged.

\section{References}

1 R. Meyer and M. Rollet, 'Barbituric Acid and Barbiturates' in 'Kirk-Othmer Encyclopedia of Chemical Technology,' Wiley, New York, 2nd Edn., 1964, p. 60.

2 D. Lednicer and L. A. Mitscher, 'The Organic Chemistry of Drug Synthesis,' Wiley, New York, 1977, p. 267.

3 D. R. Wesson and D. E. Smith, 'Barbiturates, Their Use, Misuse and Abuse,' Human Science Press, New York, 1977.

4 W. J. Doran, 'Barbituric Acid Hypnotics,' in 'Medicinal Chemistry,' eds. F. F. Blicke and H. Cox, Wiley, New York, 1959, vol. 4.

5 W. L. Nelson and L. H. Cretcher, J. Am. Chem. Soc., 1928, 50, 2758. 6 Ref. 4, Table 59, pp. 99-100.

7 M. Carrisimi, I. Grasso, E. Grumelli, E. Milla, and F. Ravenna, Farmaco, Ed. Sci., 1962, 17, 390 (Chem. Abstr., 1963, 58, 4561f).

8 S. G. Davies, 'Organotransition Metal Chemistry: Applications to Organic Synthesis,' in Organic Chemistry Series, ed. J. E. Baldwin, Pergamon, Oxford, 1982, vol. 2, p. 151.
9 M. F. Semmelhack, Ann. N.Y. Acad. Sci., 1977, 295, 36.

10 M. F. Semmelhack and H. T. Hall, J. Am. Chem. Soc., 1974, 96, 7091

11 C. C. Lee, A. S. Abd-El-Aziz, R. L. Chowdhury, A. Piórko, and R. G. Sutherland, Synth. React. Inorg. Met.-Org. Chem., 1986, 16, 541.

12 C. C. Lee, A. S. Abd-El-Aziz, R. L. Chowdhury, U. S. Gill, A. Piórko, and R. G. Sutherland, J. Organomet. Chem., 1986, 315, 79.

13 R. M. Moriarty and U. S. Gill, Organometallics, 1986, 5, 253.

14 R. G. Sutherland, A. S. Abd-El-Aziz, A. Piórko, and C. C. Lee, Synth. Commun., 1987, 17, 393.

15 A. S. Abd-El-Aziz, C. C. Lee, A. Piórko, and R. G. Sutherland, Synth. Commun., 1988, 18, 291.

16 R. A. Abramovitch, Can. J. Chem., 1958, 36, 151.

17 V. H. Wallingford, A. H. Homeyer, and D. M. Jones, J. Am. Chem. Soc., 1941, 63, 2056

18 R. G. Sutherland, M. Iqbal, and A. Piórko, J. Organomet. Chem., 1986, 302, 307.

19 R. G. Sutherland, R. L. Chowdhury, A. Piórko, and C. C. Lee, J. Organomet. Chem., 1987, 319, 379.

20 C. H. Zhang, R. L. Chowdhury, A. Piórko, C. C. Lee, and R. G. Sutherland, J. Organomet. Chem., 1988, 346, 67.

21 R. G. Sutherland, C. H. Zhang, R. L. Chowdhury, A. Piórko, and C. C. Lee, J. Organomet. Chem., 1987, 333, 367.

22 R. G. Sutherland, R. L. Chowdhury, A. Piórko, and C. C. Lee, J. Org. Chem., 1987, 52, 4618.

23 V. H. Wallingford, M. A. Thorpe, and A. H. Homeyer, J. Am. Chem. Soc., 1942, 64, 580 .

24 V. H. Wallingford, and A. H. Homeyer, U.S.P. $2391530 / 1945$ (Chem. Abstr., 1946, 40,3770 ${ }^{9}$ ).

25 V. H. Wallingford and A. H. Homeyer, U.S.P. 2478 853/1949 (Chem. Abstr., 1950, 44, 1135d).

26 E. W. Bousquet and R. Adams, J. Am. Chem. Soc., 1930, 52, 224.

27 A. Allais and M. Peterfalvi, G.P. 2163 987/1972 (Chem. Abstr., 1972, 77, 140136p).

28 J. T. Pinhey and B. A. Rowe, Tetrahedron Lett., 1980, 21, 965.

29 R. P. Kopinski, J. T. Pinhey, and B. A. Rowe, Aust. J. Chem., 1984, 37, 1245.

30 H. C. Bell, J. R. Kalman, J. T. Pinhey, and S. Sternhell, Aust. J. Chem., 1979, 32, 1521.

31 S. Ghosh, S. N. Pardo, and R. G. Salomon, J. Org. Chem., 1982, 47, 4692.

32 A. C. Knipe, S. J. McGuinness, and W. E. Watts, J. Chem. Soc., Chem. Commun., 1979, 842.

33 A. C. Knipe, S. J. McGuinness, and W. E. Watts, J. Chem. Soc., Perkin Trans. 2, 1981, 193.

34 I. U. Khand, P. L. Pauson, and W. E. Watts, J. Chem. Soc. C, 1968, 2261.

Received 13th June 1988; Paper 8/02333F 